\title{
Impact of Modern Drug Therapy on Surgery: Crohn's Disease
}

\author{
Peter Kienle \\ Department of Surgery, Theresienkrankenhaus und St. Hedwig-Klinik GmbH, Mannheim, Germany
}

Keywords

Crohn's disease - Immunosuppressive treatment . Biologicals · Surgery

\section{Summary}

Background: A significant number of patients with Crohn's disease needs to undergo surgery, and many of these patients are under modern drug treatment. These drugs may influence the rate of perioperative complications. This may have an impact on the chosen surgical strategy. Methods: This review considers clinical studies and guidelines on the effect of modern drug treatment on surgical complication rates and surgical strategy in patients with Crohn's disease. Results: Steroids above a certain dose significantly influence perioperative complications and should therefore be tapered down before performing elective surgery. Anti-TNF-alpha drugs are associated with perioperative complications, and therefore a safe interval between drug intake and surgery should be adhered to. The data on newer biologicals is scarce and conflicting; thus, it seems prudent to wait at least for one dosing interval before operating. In the emergency setting, patients undergoing bowel resection while on the above medications without an adequate interval to the last medication dose should generally get a resectional or diverting stoma. Conclusion: Modern drug treatment influences the surgical strategy in patients with Crohn's disease.
Data from this manuscript have in part been presented at the symposium 'Frontiers in Surgical Evolution' on September 21, 2018 in honor of Prof. Dr. Ernst Klar, Rostock, Germany.

\section{Introduction}

A large percentage of patients with Crohn's disease still needs to be operated during the course of their disease [1]. Over the last 30 years, the number of drugs for treating Crohn's disease has steadily increased in the therapy of inflammatory bowel diseases (IBD) (table 1) [2]. However, the rate of surgery in this patient population, if at all, has only slightly decreased in the last decades [3, 4]. The majority of these drugs interacts with the immune system and may therefore influence perioperative complication rates. This in turn may result in surgeons adopting other treatment strategies in order to prevent or minimize morbidity in this setting. Furthermore, as virtually all of the patients now are on at least one of these drugs before being scheduled for surgery, the treating surgeon should be aware of the possible negative impact such drugs may have on postoperative outcome [5]. The most important aspects that need to be considered are summarized in table 2 .

\section{Perioperative Handling of Medication}

Steroids

In numerous studies, steroids have been shown to negatively influence perioperative outcome in Crohn's disease, with a cut-off dose of around 10-20 mg prednisolone equivalent per day [6, 7]. Hence, the European Crohn's and Colitis Organisation (ECCO) and the German guidelines all recommend slow weaning of steroids in case of $20 \mathrm{mg}$ of prednisolone or equivalent if taken over a period of 6 weeks prior to elective surgery $[8,9]$. Other authors recommend slow tapering already after a period of 3 weeks of previous administration and a prednisolone equivalent of $20 \mathrm{mg}$ [5].

\section{Azathioprine}

The impact of azathioprine is less clear. Most studies actually show no effect of this drug on perioperative complications in IBD

\section{KARGER}

() 2018 S. Karger GmbH, Freiburg

Fax +497614520714
Prof. Dr. med. Peter Kienle 
Table 1. Immunosuppressive drugs with a potential impact on perioperative complications in surgery for Crohn's disease

Steroids

Azathioprine/6-mercaptopurine

Methotrexate

Cyclosporine/tacrolimus (calcineurin inhibitors)

Biologicals

Anti-TNF-alpha antagonists (infliximab, adalimumab, golimumab, etanercept, certolizumab)

Integrin antagonist (vedolizumab)

Interleukin antagonist (ustekinumab)

Table 3. Available meta-analyses on the association of anti-TNF-alpha treatment with perioperative complications in Crohn's disease

\begin{tabular}{lll}
\hline Publication, Journal & Year & $\begin{array}{l}\text { Impact of anti- } \\
\text { TNF-alpha on } \\
\text { complications }\end{array}$ \\
\hline Kopylov et al., Inflamm Bowel Dis & & \multicolumn{2}{|c}{} \\
Rosenfeld et al., J Crohns Colitis & 2012 & + \\
Billioud et al., J Crohns Colitis & 2013 & - \\
El-Hussuna et al., Dis Colon Rectum & 2013 & + \\
Narula et al., Aliment Pharmacol Ther & 2013 & $\pm^{\mathrm{a}}$ \\
Yang et al., Int J Surg & 2013 & + \\
Ahmed Ali et al., Dis Colon Rectum & 2014 & + \\
Waterland P, et al., World J Gastrointest Surg & 2014 & + \\
& 2016 & + \\
\hline
\end{tabular}

${ }^{a}$ Low-risk studies

[10]. However, upon multivariate analysis, the supposedly best study focusing on bowel resections in Crohn's disease in over 343 patients found azathioprine to be significantly associated with local septic complications [11]. As a result, the German guidelines state that this drug may also have an impact on perioperative complications in Crohn's disease, whereas the ECCO guidelines see no risk of continuing azathioprine perioperatively $[8,9]$. As azathioprine may directly interact with anesthesia, interrupting treatment for 1 day before surgery and then continuing it within 3 days after surgery seems rational. However, as the biologic duration of action of this drug overall remains unknown, stopping it completely probably only makes sense when it is not planned to be continued after surgery. In most of the cases undergoing elective surgery, azathioprine was obviously not effective in preventing surgery. Therefore, in the majority of cases it seems sensible to actually completely stop azathioprine medication when the indication for surgery has been decided on and probably as early as possible since the length of pharmacological action still is unclear.

\section{Methotrexate}

The data on methotrexate and perioperative complications in IBD is scarce. The only larger study explicitly looking at the impact of methotrexate on perioperative complications in IBD in 15 patients, 14 of these with Crohn's disease, showed no obvious correlation [12]. An additional meta-analysis in the same paper includ-
Table 2. Aspects to consider in relation to modern drug treatment in patients undergoing surgery in Crohn's disease

How to handle medication perioperatively

Continue, reduce, stop/interrupt

When to reduce or stop: safe interval

When to restart, if indicated: safe interval

\section{How to operate}

Resect or only divert

Create an anastomosis

With or without a diverting stoma

ing this cohort and four other studies on rheumatoid arthritis confirmed this result. Therefore, in conclusion, methotrexate can probably be safely continued preoperatively. It seems prudent to operate 1 week after the last dose and then restart treatment after completion of wound healing, e.g. 2 weeks after the procedure.

\section{Biologicals}

\section{Anti-TNF-Alpha}

The interpretation of the data on anti-TNF-alpha antibodies in Crohn's disease is difficult as the available studies are very heterogeneous. The interval between last drug intake (4 weeks to 6 months) and the included patient groups (surgical procedures carried out encompass small perianal procedures, mere stoma creations, and extensive large bowel resections) vary considerably, making valid meta-analyses problematic.

However, the majority of meta-analyses have indeed found an association of anti-TNF-alpha treatment (predominantly infliximab, little data on adalimumab, virtually no data on the other antiTNF-alpha antagonists) with perioperative complications (table 3 ).

Therefore, it seems very likely that there is indeed a negative impact of these 'classical' biologicals on the occurrence of perioperative complications in Crohn's disease, although there are several large series in the literature suggesting that there is no such negative association [13]. When analyzing these negative studies carefully though, they have often summarized different indications (ulcerative colitis and Crohn's disease) and procedures (subtotal colectomy, small bowel resections, stoma creation), resulting in limited statistical validity and power for the different sub-groups.

The time intervals between the last application of the biological drug vary greatly in the studies, ranging from merely 4 weeks up to 6 months, whereas in most studies, patients up to 3 months after the last drug intake were included. 6-8 weeks after the last exposure, however, there is probably not much of drug bioavailability left which can potentially affect outcome. In clinical practice, patients need different dosing intervals, reflecting the finding that serum levels may differ significantly individually. As a consequence, certain cut-off points have been determined that are associated with postoperative complications in Crohn's disease [14].

Therefore, surgery within the time of maximal drug bioavailability, e.g. just after having taken the anti-TNF antibody, should be 
avoided. The current ECCO-ESCP (European Society of ColoProctology) guidelines concede that anti-TNF therapy is associated with higher postoperative septic complications after abdominal surgery for Crohn's disease (statement 1 B) [9]. However, they conclude that the safest period for omission of the anti-TNF therapy is unknown. We would recommend waiting at least as long as the individual dosing interval of the biological being taken before performing elective surgery. This means that patients on infliximab should not be operated prior to 4 weeks after having taken the last dose, while patients on adalimumab should wait at least 2 weeks, ideally 3-4 weeks as the dosing interval generally is only 2 weeks, but the half-life of the drug is considerably longer.

Due to pragmatic reasons, we generally discontinue the treatment at least 4 weeks prior to elective surgery, which is also the recommendation from other high-volume centers [5].

Treatment can be restarted earliest after completion of wound healing, e.g. 2 weeks after the procedure. However, we would generally recommend waiting 3-4 weeks as this would make sure that the anastomosis has definitely healed, and a real benefit of starting anti-TNF-alpha earlier than that has not been shown and is unlikely.

\section{Anti-Integrin Antagonist (Vedolizumab)}

Up until now, there is little data on this drug regarding perioperative complications as approval by the Food and Drug Administration (FDA) was granted only 4 years ago. Due to the mode of action as a selective integrin inhibitor in the gut, there is concern that anastomotic healing after bowel resection may be impaired [15].

In the first large comparative study in patients with IBD (approximately three-fourths with Crohn's disease), Lightner et al. [16] found a significantly increased postoperative surgical site infection (SSI) rate in the group having received vedolizumab within 12 weeks prior to surgery compared to a control group under antiTNF-alpha antagonists and a control group without medication (36 vs. 10 vs. $13 \%, \mathrm{p}<0.001$ ). On multivariate analysis, vedolizumab remained a significant predictor of postoperative SSIs $(\mathrm{p}<$ 0.001). However, this study has several limitations. Patients in the vedolizumab group had much higher C-reactive protein levels (about 60 vs. $10 \mathrm{mg} / \mathrm{l}$ ), were significantly younger, and had significantly more additional immunomodulators in their treatment. Moreover, $74 \%$ of patients in this group did not undergo primary anastomosis or additionally received a diverting stoma compared to 66 and $41 \%$ of patients in the other groups, respectively. All this insinuates that the vedolizumab group was a negatively selected group of patients with an especially severe course of IBD, which is reflected by the following statement in the paper: 'many of the patients initially treated with vedolizumab were patients with medically refractory disease awaiting its FDA approval'. In conclusion, the risk of a negative selection bias is very likely in this analysis.

A smaller study, only published as an abstract, looked at 48 patients undergoing right hemicolectomy for Crohn's disease subclassified into 4 groups (vedolizumab, anti-TNF-alpha antagonists, steroids, no medication) and found no difference in complications
[17]. Due to the small numbers (12 per group), however, the statistical power of this analysis is low.

In conclusion, there is still insufficient data to adequately make any statement in regard to vedolizumab and perioperative complication rates. Therefore, it seems prudent to at least wait as long as the individual dosing interval, meaning that this drug should be discontinued at least 8 weeks prior to surgery. Treatment can be restarted earliest after completion of wound healing, e.g. 2 weeks after the procedure. Yet again, like in the case of anti-TNF-alpha treatment, we would generally recommend waiting 3-4 weeks as this would ensure that the anastomosis has definitely healed, and a real benefit of starting vedolizumab earlier is unlikely.

\section{Ustekinumab}

As ustekinumab has only recently been approved by the FDA, up until now only a single study has analyzed perioperative complication rates in comparison to patients treated with anti-TNF-alpha antagonists [18]. Although SSIs were not significantly different in the two groups, there was a significantly higher revision rate in the ustekinumab group (16 vs. $4 \%$ ). The anastomotic leak rate was also higher (9 vs. 3\%), albeit not significantly. When taking into account that only around $50 \%$ of patients in the ustekinumab group actually underwent construction of an anastomosis versus $>75 \%$ in the anti-TNF group, suggesting a possible selection bias, these results raise some concern.

In conclusion, there is still insufficient data if ustekinumab influences perioperative complication rates. Therefore, it seems prudent to at least wait as long as the individual dosing interval, meaning that this drug should be discontinued at least 8 weeks prior to surgery. Treatment can be restarted earliest after completion of wound healing, e.g. 2 weeks after the procedure. Yet again, like in the case of anti-TNF-alpha treatment, we would generally recommend waiting 3-4 weeks as this would ensure that the anastomosis has definitely healed, and a real benefit of starting ustekinumab earlier is unlikely.

\section{Surgical Strategy: How to Operate}

\section{Elective Setting}

If patients can be weaned of steroids below a dose of $<20 \mathrm{mg}$ prednisolone equivalent (better: $\leq 10 \mathrm{mg}$ ), then surgery can be performed as if the patient is not under any immunosuppression [9]. If this is not possible, the creation of a stoma should rather be the rule than the exception, especially if there are other risk factors such as a poor nutritional status. Either the bowel ends should then be exteriorized as a resectional stoma or a created anastomosis should be protected by a diverting stoma. Azathioprine and calcineurin inhibitors (tacrolimus) alone have no influence on the choice of surgical strategy. Only if given in combination with other drugs such as anti-TNF-alpha, these drugs may influence the surgical strategy in the direction of a more conservative approach, e.g. by not performing an anastomosis or, if doing so protecting it, by a diverting stoma. If patients are under biologicals and the operation 
cannot be postponed until a safe interval is reached (anti-TNF-alpha 4 weeks, vedolizumab and ustekinumab 8 weeks), which is very rarely the case in an elective setting, again a conservative approach is sensible, e.g. construction of a resectional stoma or at least a diverting stoma. This concept is also endorsed by the current German and ECCO-ESCP guidelines [8, 9].

\section{Emergency Setting}

If the patient needs to be operated in an emergency, e.g. acute ileus or perforation, then the emergency setting in itself already warrants a conservative approach, e.g. a primary anastomosis should only be done in patients without other risk factors such as bad nutritional status etc. If the patient additionally is under a higher dose of prednisolone equivalent ( $\geq 20 \mathrm{mg}$ ) or under biologicals without being in the safe interval (see also sections on the specific biological drug above), creation of a stoma, either as a resectional or as a diverting stoma, is urgently recommended [8].

\section{Disclosure Statement}

The author does not have any conflicts of interest to declare.

\section{References}

1 Bouguen G, Peyrin-Biroulet L: Surgery for adult Crohn's disease: what is the actual risk? Gut 2011;60: $1178-1181$.

2 Reinglas J, Gonczi L, Kurt Z, et al: Positioning of old and new biologicals and small molecules. World J Gastroenterol 2018;24:3567-3582.

3 Burke JP, Velupillai Y, Ronan O'Connell PR, Coffey JC: National trends in intestinal resection for Crohn's disease in the post-biologic era. Int J Colorectal Dis 2013;28:1401-1406.

4 deBruyn JCC, Shian Soon I, Hubbard J, et al: Nationwide temporal trends in incidence of hospitalization and surgical intestinal resection in pediatric inflammatory bowel diseases in the United States from 1997 to 2009. Inflamm Bowel Dis 2013;19:2423-2432.

5 Lightner A, Shen B: Perioperative use of immunosuppressive medications in patients with Crohn's disease in the new 'biological era'. Gastroenterol Rep (Oxf) 2017;5:165-177.

- 6 Huang W, Tang Y, Nong L, Sun Y: Risk factors for postoperative intra-abdominal septic complications after surgery in Crohn's disease: a meta-analysis of observational studies. J Crohns Colitis 2015;9:293-301.
7 Subramanian V, Saxena S, Kang JY, Pollok RC: Preoperative steroid use and risk of postoperative complications in patients with inflammatory bowel disease undergoing abdominal surgery. Am J Gastroenterol 2008; 103:2373-2381.

8 Preiß JC, Bokemeyer B, Buhr HJ: Updated German clinical practice guideline on 'Diagnosis and treatment of Crohn's disease (Article in German). Z Gastroenterol 2014;52:1431-1484.

$\checkmark$ Bemelman WA, Warusavitarne J, Sampietro GM, et al: ECCO-ESCP consensus on surgery for Crohn's disease. J Crohns Colitis 2018;12:1-16.

10 Aberra FN, Lewis JD, Hass D, et al: Corticosteroids and immunomodulators: postoperative infectious complication risk in inflammatory bowel disease patients. Gastroenterology 2003;125:320-327.

11 Myrelid P, Olaison G, Sjödahl R, et al: Thiopurine therapy is associated with postoperative intra-abdominal septic complications in abdominal surgery for Crohn's disease. Dis Colon Rectum 2009;52:13871394.

12 Afzali A, Park C, Zhu K, et al: Preoperative use of methotrexate and the risk of early postoperative complications in patients with inflammatory bowel disease. Inflamm Bowel Dis 2016;22:1887-1895.
13 Waterman M, Xu W, Dinani A, et al: Preoperative biological therapy and short-term outcomes of abdominal surgery in patients with inflammatory bowel disease. Gut 2013;62:387-394

14 Lau C, Dubinsky M, Melmed G, et al: The impact of preoperative serum anti-TNFa therapy levels on early postoperative outcomes in inflammatory bowel disease surgery. Ann Surg 2015;261:487-496.

15 Wyant T, Fedyk E, Abhyankar B: An overview of the mechanism of action of the monoclonal antibody vedolizumab. J Crohns Colitis 2016;10:1437-1444.

16 Lightner AL, Raffals LE, Mathis KL, et al: Postoperative outcomes in vedolizumab-treated patients undergoing abdominal operations for inflammatory bowel disease. J Crohns Colitis 2017;11:185-190.

17 Schils N, De Buck van Overstraeten A, Vermeire S, et al: P445 Perioperative use of vedolizumab seems not associated with short-term-postoperative complications in patients with Crohn's disease undergoing right hemicolectomy with ileocolonic anastomosis. J Crohns Colitis 2017;11(suppl 1):S304.

18 Lightner AL, McKenna NP, Tse CS, et al: Postoperative outcomes in ustekinumab-treated patients undergoing abdominal operations for Crohn's disease. J Crohn's Colitis 2018;12:402-407. 